\title{
Diseño, implementación y evaluación de un módulo basado en el modelo RAMM para la enseñanza del concepto de constitución en un curso de química orgánica escolar
}

Ruth Esmeralda Sánchez Sánchez ${ }^{1}$

ruthesmeralda@gmail.com

\section{Resumen}

El presente documento esboza el proceso investigativo de práctica pedagógica y didáctica que reconoce los modelos moleculares y las fórmulas estructurales como las principales herramientas conceptuales, teóricas y metodológicas en la construcción de saber alrededor de la estructura de las sustancias, y que parte de una reflexión histórica acerca de los conceptos moleculares en Química. Desde esta perspectiva, se implementó un módulo basado en el modelo RAMM, como un instrumento didáctico para la enseñanza de los conceptos de Química orgánica, como la isomería, convirtiendo los procesos de aula en espacios de discusión.

\section{Palabras clave}

Metodología RAMM, Fórmulas Estructurales, Isomería Topológica, Enseñanza, Aprendizaje 


\section{Introducción}

En el problema estructura de las sustancias, se ve limitado el manejo único de fórmulas químicas y es relevante el avance hacia las formulaciones estructurales para resolver problemas como el de los isómeros topológicos, también llamados isómeros constitucionales; donde la relación de conectividad hace serias diferencias entre una y otra sustancia que, aunque presentan los mismos átomos y en la misma proporción, tienen una distribución espacial diferente, lo que hace que cambien sus propiedades (Jensen, 1998), haciendo necesario el manejo de las fórmulas estructurales y modelos moleculares que funcionen como herramientas didácticas para la enseñanza de conceptos y teorías químicas.

Partiendo de la metodología de Registro de Análisis de Modelos Moleculares -RAMM- y de los referentes curriculares establecidos para el área de Química, se delimitan unas condiciones iniciales para plantear un modelo didáctico de enseñanza, que permita al estudiante reconocer problemas que lo lleven a solucionar dificultades en el aula. El estudiante debe encontrarse con problemas de orden composicional, constitucional (trabajando parcialmente la metodología RAMM), debe reconocer los límites de cada uno de estos, es decir, a qué elementos debe recurrir para enfrentar diferentes eventos que se encuentran en las estructuras de la sustancia y cómo cada uno de estos conceptos se pueden trasladar a la construcción de otras concepciones, como lo es la isomería topológica.

Los modelos teóricos de la estructura molecular surgen, con mucha frecuencia, en los problemas de reflexión didáctica, en el cerco de la investigación y en la producción académica alrededor de la enseñanza de la Química. En este sentido, la implementación de los modelos moleculares en el aula promovería una nueva etapa para el aprendizaje de la Química, al ubicarse como una herramienta con la que se puede abordar la enseñanza de los conceptos y teorías químicas, que se fortalecen desde la estructura de las sustancias, articulando temáticas tales como el problema del enlace químico, la conformación de las moléculas, las propiedades químicas derivadas de la estructura molecular, entre otras. Esto hace necesario su papel en la innovación de las prácticas didácticas y abre espacios en los currículos de la escuela, reconociendo los Modelos Moleculares y las fórmulas estructurales como herramientas discursivas y metodológicas, que puedan ser utilizadas para la reflexión y discusión, al abordar concepciones y teorías derivadas de la estructura molecular para la resolución de problemas en el ámbito escolar.

Se denota así su importancia en la renovación de prácticas didácticas, por medio del la Metodología Registro de Análisis de Modelos Moleculares -RAMM-, consolidando este trabajo como un aporte en el proceso de formación en Química de los estudiantes de educación básica y media, además de dejar preocupaciones a los actores del proceso, es decir, a los estudiantes y profesores en formación y en ejercicio.

\section{La historia de los modelos moleculares como una herramienta discursiva}

La teoría atómica de Dalton abrió el camino para el afianzamiento de una química disciplinaria, de un campo de conocimiento paradigmático, alrededor del problema de la sustancialidad de la materia, su estructura y sus interacciones. El proyecto berzeliusiano concretó en la teoría de las fórmulas una poderosa herramienta de aproximación a la diversidad material de las sustancias químicas, partiendo de dos principios fundamentales: la identidad de los materiales en tanto cuerpos homogéneos y unitarios, diferenciables unos de otros por sus propiedades físicas y químicas, y la unidad por su composición elemental única y una distribución entero-numérica irrepetible (Martínez, A.S. 2005), viéndose alterado por la emergencia de los problemas 
isoméricos y la extraña disposición de los compuestos orgánicos, que oponían resistencia a los tratamientos teóricos aceptados.

En ese momento, la explicación de las propiedades químicas de las sustancias no podía derivarse de la consideración de los elementos que los componen ni de su relación entero-numérica, porque surgirían como problemáticas principales, tanto la distribución espacial de los átomos, como la configuración estructural en las moléculas. August Kekulé puso de manifiesto el carácter geométrico de la estructuración de los átomos en el espacio y su incidencia en las propiedades químicas y físicas de las sustancias, siendo estas las matrices a de problemática definida (Del Re, G. 1998). La estructura molecular como concepto se reconoció con mayor contundencia a partir de la determinación de las estructuras isómeras del benceno, en sus derivados polisustituidos y la explicación de sus diferencias a partir de argumentos estructurales, teniendo en cuenta el estudio de la geometría de las moléculas y la estructura de los materiales y, como consecuencia, la ascensión de modelos de visualización de estructuras moleculares de las sustancias, concretando al modelado molecular como una práctica conceptual y experimental que da cuenta de la estructura y propiedades de la sustancialidad.

\section{La metodología de registro de análisis de modelos moleculares -RAMM-}

El análisis estructural, la construcción de conceptos y teorías alrededor del problema de la estructura de las sustancias, al igual que las herramientas y escenarios experimentales para su constitución fenoménica están íntima y sólidamente ligados al desarrollo de los modelos moleculares. Una historia de las prácticas de modelado molecular, en los diversos momentos de consolidación de la disciplina química, muestra el camino de realización discursiva acerca de la estructuración de la sustancialidad material y sus propiedades, siendo el espacio propicio para evidenciar las rupturas y discontinuidades epistemológicas, las transformaciones conceptuales, la emergencia de nuevos problemas y la aparición de modos de producción de conocimiento; evidenciando la intención de construir un discurso didáctico alrededor de los estudios estructurales, la proposición y validación de estrategias metodológicas para el abordaje de análisis acerca de la estructura de las sustancias en química, en los espacios de enseñanza.

El propósito de la metodología de Registro de Análisis de Modelos Moleculares -RAMM- vincula problemas históricos, epistemológicos y didácticos para la propuesta de ejercicios prácticos de análisis estructural en Química. La metodología RAMM considera que los modelos moleculares son herramientas conceptuales y metodológicas para la investigación estructural en Química. De este modo, se propone el estudio de cuatro aspectos fundamentales de las moléculas, a través de la manipulación de modelos moleculares: la composición, la constitución, la configuración y la conformación. Estos cuatro elementos se derivan del concepto didáctico de molécula, construido mediante NIP, que señala que la molécula, como unidad estructural fundamental de las sustancias, se caracteriza por estos cuatro componentes conceptuales (Martínez, A.S. 2004). Este estudio se recoge por medio de un registro escrito, en el que se deben explicar las propiedades composicionales, constitucionales, configuracionales

August Kekulé puso de manifiesto el carácter geométrico de la estructuración de los átomos en el espacio y su incidencia en las propiedades químicas y físicas de las sustancias, siendo estas las matrices a de problemática definida (Del Re, G. 1998). 
y conformacionales, desde los conceptos de la estructura molecular propuestos. Partiendo de la descripción y de la comparación de diferentes especies moleculares y sus elementos, en este documento de estudio estructural se recopila la información general de la molécula, así como sus aspectos de composición, configuración y conformación, fundamentales para los estudios estructurales en Química. Incluso, sirven de soporte teórico para los hallazgos experimentales de simetría y asimetría.

\section{Diseño del módulo de trabajo}

Se realizó en tres etapas. Primero, se llevó a cabo la revisión conceptual acerca de la metodología RAMM, isómeros topológicos y la enseñanza de la Química, alrededor de la problemática de la estructura de las sustancias. Para la segunda etapa, se construyó una prueba piloto basada en el concepto de constitución en relación con el concepto de composición; esta prueba se aplicó a diez estudiantes de grado undécimo. En un tercer momento, el análisis de resultados se hizo teniendo en cuenta la recurrencia del estudiante en la utilización de fórmulas moleculares o estructurales, al enfrentarse con problemas de orden composicional y constitucional. Finalmente, el módulo se elaboró con base en la metodología RAMM, teniendo en cuenta los aspectos de composición y constitución. Los conceptos de configuración y conformación no fueron utilizados para la realización del módulo, ya que se trabajaría isomería constitucional o topológica, en moléculas orgánicas.

\section{Implementación del módulo de trabajo}

Se comenzó con un avance generalizado delos aspectos estructurales de una molécula, trabajados desde la metodología RAMM, pero de modo parcial, ya que solo se tuvieron en cuenta los aspectos generales, la composición y la constitución. La composición se estudia a partir de la caracterización del concepto de fórmula (relativa- absoluta) y la verificación de las leyes ponderales en la nominación y clasificación de las sustancias (Jensen, 1998); luego, se tuvo en cuenta la constitución, recurriendo a las fórmulas estructurales y describiendo la conexión de los átomos en el espacio. Para la realización de este trabajo se emplearon sesiones de seminarios de investigación alemán, espacio de discusión y reflexión de las lecturas propuestas; talleres de modelado molecular, donde se modelan las estructuras de las sustancias con ayuda del equipo de esferas y bastones de casquete perforado y se elaboran estudios estructurales comparativos.

\section{Evaluación de los aprendizajes}

El análisis se obtuvo teniendo en cuenta las diferentes actividades realizadas durante el proceso de implementación del módulo, pruebas como la tipo Likert, de recurrencia, entre otras, que ayudan a tener una aproximación del desempeño obtenido y la evolución del espacio de formación durante la aplicación del módulo.

\section{Resultados}

Conceptualización: se hace una aproximación a las concepciones de los estudiantes acerca de las relaciones entre Química, Sustancia, Molécula. Para la primera categoría, los resultados arrojan un relativo conocimiento en la relación epistemológica del objeto de estudio de la Química; para las siguientes categorías se nota la confusión en las relaciones de orden conceptual.

Documento RAMM Parcial: se aproxima a las concepciones de los estudiantes acerca de los aspectos composicionales y constitucionales de las sustancias y su relación con las propiedades molares y moleculares. De acuerdo con los resultados obtenidos durante el proceso, se 
determina que los estudiantes tienen un relativo reconocimiento de las propiedades molares y las relacionan con los aspectos generales y composicionales de las sustancias, con lo que se observa la recurrencia de la descripción de las sustancias desde propiedades organolépticas y que conocen su composición por porcentaje de masas y pesos. Para la categoría de reconocimiento de propiedades moleculares se encuentran dificultades en el momento de establecer relaciones de estas propiedades con las estructuras moleculares (uso de fórmulas estructurales) y la forma en que los átomos se encuentran conectados en el espacio (topología de enlace).

Pruebas tipo Likert 1 y 2: Se puede evidenciar el proceso de los estudiantes al conocer sus concepciones acerca de la composición y constitución de las sustancias. Reforzaron las relaciones molares con la composición y las propiedades organolépticas de las sustancias; presentan dificultades con las relaciones moleculares y con el uso de las fórmulas estructurales. De la primera a la segunda prueba, los estudiantes no muestran indiferencia, sino que, por el contrario, toman posición, aproximándose a las discusiones disciplinares acerca de la problemática de la estructura de las sustancias.

\section{Conclusión}

Las fórmulas estructurales y los Modelos Moleculares son las principales herramientas conceptuales, teóricas y metodológicas en los análisis constitucionales de isómeros topológicos, así como la metodología de Registro de Análisis de Modelado Molecular -RAMM-; se reconocen como un instrumento discursivo y metodológico para la reflexión y discusión de concepciones y teorías acerca de la estructura molecular, para la resolución de problemas en la escuela.

El diseño, la implementación y la evaluación del módulo basado en la metodología RAMM, para el estudio de conceptos en Química orgánica alrededor de la estructura de las sustancias, se consolida como una aproximación curricular para la transformación de concepciones y la evolución discursiva de los actores que intervienen en el proceso. De este modo, concibiendo la práctica pedagógica dentro de la modalidad de innovaciones por medio del Modelado Molecular, este trabajo se consolida como un aporte en el proceso de formación en Química de los estudiantes de educación básica y media, además de dejar preocupaciones a estudiantes y profesores en formación y en ejercicio.

\section{Bibliografía}

Del Re, G. (1998). Ontological status of Molecular Structure. HYLE-International Journal for Philosophy of Chemistry. 4(1), 81-103.

Grupo de investigación Pensamiento, Ciencia y Enseñanza (2005). Proyecto de Investigación. La Enseñanza de los Modelos teóricos de la Estructura Molecular DQU-040-04. Informe Final. Bogotá: Universidad Pedagógica.

Jensen, W. B. (1998). A logic, history and the chemistry textbooks 1: Does chemistry have a logical structure?. Journal of Chemical Education, 75(6), 679-687.

Martínez, A.S. (2004). Estudio estructural de la molécula de Ciclohexano. Esferas \& Bastones, 2(1), 3-10.

Martínez, A.S. (2005). Aproximación al concepto de alómero. Esferas \& Bastones, 3(1), 4-11. 